\title{
Distributed Lagrange Multiplier Method for Particulate Flows with Collisions
}

\author{
P. Singh* \\ Department of Mechanical Engineering \\ New Jersey Institute of Technology \\ University Heights, Newark, New Jersey 07102-1982 \\ Phone: (973)596-3326, Fax: (973)642-4282 \\ singhp@njit.edu \\ T.I. Hesla and D.D. Joseph \\ Department of Aerospace Engineering and Mechanics \\ University of Minnesota \\ 107 Akerman Hall, 110 Union St SE, Minneapolis, MN 55455 \\ joseph@aem.umn.edu
}

Keywords: Particulate Flows, finite element method, direct numerical simulations, viscoelastic fluid, Oldroyd-B fluid, particle collisions.

A modified distributed Lagrange multiplier/fictitious domain method (DLM) that allows particles to undergo collisions is developed for particulate flows. In the earlier versions of the DLM method for Newtonian and viscoelastic liquids described in Glowinski, Pan, Hesla, Joseph (1998) and Singh, Joseph, Hesla, Glowinski, Pan (2000) the particle surfaces were restricted to be at least one and a half times the velocity element size away from each other. A repulsive body force was applied to the particles when the distance between them was smaller than this critical value. This was necessary for ensuring that conflicting rigid body motion constraints from two different particles are not imposed at the same velocity nodes.

In the modified DLM method the particles are allowed to come arbitrarily close to each other and even slightly overlap each other. When conflicting rigid body motion constraints from two different particles are applicable on a velocity node, the constraint from the particle that is closer to that node is used and the other constraint is dropped. An elastic repulsive force is applied when the particles overlap each other. In our simulations, the particles are allowed to overlap as much as one hundredth of the velocity element size. The modified DLM method is implemented for both Newtonian and viscoelastic liquids.

Our simulations show that when particles are dropped in a channel, and the viscoelastic Mach number (M) is less than one and the elasticity number $(\mathrm{E})$ is greater than one, the particles form a chain parallel to the flow direction. As in experiments, the new method allows particles in the chain to approximately touch each other. The particles dropped in a Newtonian liquid, on the other hand, undergo characteristic drafting, kissing and tumbling. During the touching phase, as in experiments, the two particles touch each other. The modified method thus allows hydrodynamic forces to be fully 
resolved to within the tolerance of the mesh and thus the extra artificial force in a security zone outside the particle which are used in all other methods are not needed.

\section{Introduction}

Collisions-or near-collisions-between particles present severe difficulties in direct simulations of particulate flows. In point of fact, however, smooth particles cannot actually contact each other in finite time in the continuous system (also see Leal 1992). In light of this, the term "collision" is somewhat misleading - at least for smooth particles. However, since the gap width can become exceedingly small in certain situations, numerical truncation errors may allow actual contact (or even overlap) to occur in simulations.

Even near-collisions can greatly increase the cost of a simulation, because in order to simulate the particle-particle interaction mechanism, the flow fields in the narrow gap between the converging particle surfaces must be accurately resolved. The element size required for this decreases with the gap width, leading to extremely small elements and greatly increased numbers of unknowns to be solved for. Actual overlap can lead to overdetermined systems of equations, and must thus be prevented at all costs.

In numerical simulations to date (Glowinski, Pan, Hesla, Joseph 1998, Singh, Joseph, Hesla, Glowinski, Pan 2000, Huang, Hu, Joseph 1998, Hu 1996, Huang, Feng, Hu, Joseph 1997, Glowinski, Pan, Periaux 1997, Girault, Glowinski, Pan 1999), this problem is avoided by introducing an artificial repulsive force between particles which keeps the particle surfaces more than one element apart from each other. In the standard distributed Lagrange multiplier/fictitious domain (DLM) method (Glowinski et al 1998, Singh et al 2000, Glowinski et al 1997, Girault et al 1999), this ensures that the differing rigid-body motion constraints of the two particles are not simultaneously imposed at any one velocity node, which would lead to a numerically overdetermined system of equations. In the arbitrary Lagrangian-Eulerian (ALE) method (Huang et al 1998, Hu 1996, Huang et al 1997), it ensures that the element size doesn't become excessively small. The ALE method requires two layers of elements in the gap between the converging surfaces.

In this paper, we present a modification of the DLM finite-element scheme described in (Glowinski et al 1998, Singh et al 2000) which allows particles to come arbitrarily close to each other, and even overlap slightly. The basic idea, which is due to D.D. Joseph, is to simply drop one of the conflicting rigid-body motion constraint terms in the equation of motion-the one corresponding to the particle surface which is farther away from the node in question. This trick avoids overdetermined systems of equations, but is applicable only as long as the overlap of the particles does not exceed one element. An elastic repulsive force is introduced to prevent particles from overlapping more than that.

\footnotetext{
* Corresponding author.
} 
The modified DLM method is verified by comparing the time dependent trajectories for two circular particles falling in a channel for two different mesh refinements, and for two different time steps. It is shown that the results are independent of the mesh resolution and the time step.

It was discussed in (Glowinski et al 1998, Singh et al 2000, Fortes, Joseph, Lundgren 1987, Joseph, Fortes, Lundgren, Singh 1987) that when two or more particles are dropped in a channel filled with a viscoelastic liquid the orientation of particles relative to the direction of flow is determined by the viscoelastic Mach number $\mathrm{M}=\sqrt{\operatorname{Re} \mathrm{De}}$ and the elasticity number $\mathrm{E}=\mathrm{De} / \mathrm{Re}$. Here $\mathrm{De}=\mathrm{U} \lambda_{\mathrm{r}} / \mathrm{D}$ is the Deborah number, and $\operatorname{Re}=\rho_{\mathrm{L}} \mathrm{UD} / \eta$ is the Reynolds number, where $\lambda_{\mathrm{r}}$ is the relaxation time of the fluid, $\eta$ is the zero shear viscosity of the fluid, $U$ is the particle velocity, and $D$ is the particle diameter. Specifically, when $\mathrm{M}<1$ and $\mathrm{E}>1$, the particles align themselves parallel to the flow direction. The distance between the particles of a chain may be very small. In fact, the particles may even approximately touch each other. On the other hand, when these conditions are not satisfied, or when the fluid is Newtonian, the particles undergo drafting, kissing and tumbling. Experiments show that during kissing the particles come very close to each other, and may even approximately touch each other.

In the next section the strong and weak forms of governing equations as well as the modified DLM scheme that allows particle-particle near collisions is presented. In the last section, we present several test cases to validate the scheme.

\section{Computational Scheme}

The computational scheme we propose is a modification of the DLM finite-element scheme described in Glowinski et al (1998) and Singh et al (2000). In this scheme, the fluid flow equations are solved on the combined fluid-solid domain, and the motion inside the particle boundaries is forced to be rigid-body motion using a distributed Lagrange multiplier. The fluid and particle equations of motion are combined into a single combined weak equation of motion, eliminating the hydrodynamic forces and torques, which helps ensure stability of the time integration. The time integration is performed using the Marchuk-Yanenko operator splitting method, which is first-order accurate. See Glowinski et al (1998) and Singh et al (2000) for further details.

\subsection{Governing Equations}

In this paper we will present results for two-dimensional flows. Let us denote the domain containing the Newtonian or viscoelastic fluid and $\mathrm{N}$ particles by $\Omega$, and the interior of the ith particle by $P_{i}(t)$. For simplicity we will assume that the domain is rectangular with boundary $\Gamma$. The four sides 
of the domain will be denoted by $\Gamma_{1}, \Gamma_{2}, \Gamma_{3}$, and $\Gamma_{4}$ (see Fig. 1), and $\Gamma^{-}$will be used to denote the upstream part of $\Gamma$. The viscoelastic fluid is modeled by the Oldroyd-B model. The governing equations for the fluid-particle system are:

$$
\begin{array}{ll}
\rho_{\mathrm{L}}\left[\frac{\partial \mathbf{u}}{\partial \mathrm{t}}+\mathbf{u} \cdot \nabla \mathbf{u}\right]=\rho_{\mathrm{L}} \mathbf{g}-\nabla \mathrm{p}+\nabla \cdot\left(\frac{\mathrm{c}}{\lambda_{\mathrm{r}}} \mathbf{A}\right)+\nabla \cdot\left(2 \eta_{\mathrm{s}} \mathbf{D}\right) & \text { in } \Omega \backslash \overline{\mathrm{P}(\mathrm{t})} \\
\nabla \cdot \mathbf{u}=0 & \text { in } \Omega \backslash \overline{\mathrm{P}(\mathrm{t})} \\
\mathbf{u}=\mathbf{u}_{\mathrm{L}} & \text { on } \Gamma \\
\mathbf{u}=\mathbf{U}_{\mathrm{i}}+\omega_{\mathrm{i}} \times \mathbf{r}_{\mathrm{i}} & \text { on } \partial \mathrm{P}_{\mathrm{i}}(\mathrm{t}), \mathrm{i}=1, \ldots, \mathrm{N},
\end{array}
$$

with the evolution of the configuration tensor $\mathbf{A}$ given by

$$
\frac{\partial \mathbf{A}}{\partial \mathbf{t}}+\mathbf{u} \cdot \nabla \mathbf{A}=\mathbf{A} \cdot \nabla \mathbf{u}+\nabla \mathbf{u}^{\mathrm{T}} \cdot \mathbf{A}-\frac{1}{\lambda_{\mathrm{r}}}(\mathbf{A}-\mathbf{I}),
$$

$$
\mathbf{A}=\mathbf{A}_{\mathrm{L}} \quad \text { on } \Gamma^{-} .
$$

Here $\mathbf{u}$ is the velocity, $p$ is the pressure, $\eta_{\mathrm{s}}$ is the solvent viscosity, $\rho_{\mathrm{L}}$ is the fluid density, $\mathbf{D}$ is the symmetric part of the velocity gradient tensor, $\mathrm{c}$ is a measure of polymer concentration in terms of the zero shear viscosity, and where $\lambda_{\mathrm{r}}$ is the relaxation time. The zero shear viscosity $\eta=\eta_{\mathrm{s}}+\eta_{\mathrm{p}}$, where $\eta_{\mathrm{p}}=\mathrm{c} \eta_{\mathrm{s}}$ is the polymer contribution to viscosity. The fluid retardation time is equal to $\lambda_{\mathrm{r}} /(1+\mathrm{c})$. The above equations are solved with the following initial conditions:

$$
\begin{aligned}
& \left.\mathbf{u}\right|_{\mathrm{t}=0}=\mathbf{u}_{0} \\
& \left.\mathbf{A}\right|_{\mathrm{t}=0}=\mathbf{A}_{0}
\end{aligned}
$$

where $\mathbf{u}_{0}$ and $\mathbf{A}_{0}$ are the known initial values of the velocity and the configuration tensor. The particle velocity $\mathbf{U}_{\mathrm{i}}$ and angular velocity $\omega_{\mathrm{i}}$ are governed by

$$
\begin{aligned}
& \mathbf{M}_{\mathrm{i}} \frac{\mathrm{d} \mathbf{U}_{\mathrm{i}}}{\mathrm{dt}}=\mathbf{M}_{\mathrm{i}} \mathbf{g}+\mathbf{F}_{\mathbf{i}} \\
& \mathrm{I}_{\mathrm{i}} \frac{\mathrm{d} \omega_{\mathrm{i}}}{\mathrm{dt}}=\mathrm{T}_{\mathrm{i}} \\
& \left.\mathbf{U}_{\mathrm{i}}\right|_{\mathrm{t}=0}=\mathbf{U}_{\mathrm{i}, 0} \\
& \left.\omega_{\mathrm{i}}\right|_{\mathrm{t}=0}=\omega_{\mathrm{i}, 0}
\end{aligned}
$$

where $M_{i}$ and $I_{i}$ are the mass and moment of inertia of the ith particle, and $\mathbf{F}_{i}$ and $\mathbf{T}_{\mathrm{i}}$ are the hydrodynamic force and torque acting on the ith particle. In this investigation we will assume that the particles are circular, and therefore we do not need to keep track of the particle orientation. The particle positions are obtained from 


$$
\begin{aligned}
& \frac{\mathrm{d} \mathbf{X}_{\mathrm{i}}}{\mathrm{dt}}=\mathbf{U}_{\mathrm{i}} \\
& \left.\mathbf{X}_{\mathrm{i}}\right|_{\mathrm{t}=0}=\mathbf{X}_{\mathrm{i}, 0}
\end{aligned}
$$

where $X_{i, 0}$ is the position of the ith particle at time $t=0$.

\subsection{Weak form of equations and finite-element discretization}

The approach used for obtaining the weak form of the governing equations stated in the previous section was described in Glowinski et al (1998), Singh et al (2000) and Glowinski et al (1997). In obtaining this weak form, the hydrodynamic forces and torques acting on the particles can be completely eliminated by combining the fluid and particle equations of motion into a single weak equation of motion for the combined fluid-particle system. For simplicity, in this section we will assume that there is only one particle. The extension to the many-particle case is straightforward.

The solution and variation are required to satisfy the strong form of the constraint of rigid body motion throughout $\mathrm{P}(\mathrm{t})$. In the distributed Lagrange multiplier method this constraint is removed from the velocity space and enforced weakly as a side constraint using a distributed Lagrange multiplier term. It was shown in Glowinski et al (1998) and Glowinski et al (1997) that the following weak formulation of the problem holds in the extended domain:

For a.e. $\mathrm{t}>0$, find $\mathbf{u} \in \overline{\mathrm{W}}_{\mathrm{u} \Gamma}, \mathbf{A} \in \overline{\mathrm{W}}_{\mathrm{A}}, \mathrm{p} \in \mathrm{L}_{0}^{2}(\Omega), \lambda \in \Lambda(\mathrm{t}), \mathbf{U} \in \mathbf{R}^{2}$, and $\omega \in \mathbf{R}$, satisfying

$$
\begin{aligned}
& \int_{\Omega} \rho_{\mathrm{L}}\left(\frac{\mathrm{d} \mathbf{u}}{\mathrm{dt}}-\mathbf{g}\right) \cdot \mathbf{v} \mathrm{d} \mathbf{x}-\int_{\Omega} \mathrm{p} \nabla \cdot \mathbf{v} \mathrm{d} \mathbf{x}+\int_{\Omega} 2 \eta_{\mathrm{s}} \mathbf{D}[\mathbf{u}]: \mathbf{D}[\mathbf{v}] \mathrm{d} \mathbf{x}-\int_{\Omega} \mathbf{v} \cdot \nabla \cdot\left(\frac{\mathrm{c}}{\lambda_{\mathrm{r}}} \mathbf{A}\right) \mathrm{d} \mathbf{x} \\
& +\left(1-\frac{\rho_{\mathrm{L}}}{\rho_{\mathrm{d}}}\right)\left(\mathrm{M}\left(\frac{\mathrm{d} \mathbf{U}}{\mathrm{dt}}-\mathbf{g}\right) \cdot \mathbf{V}+\mathrm{I} \frac{\mathrm{d} \omega}{\mathrm{dt}} \xi\right)-\mathbf{F}^{\prime} \cdot \mathbf{V}=\langle\boldsymbol{\lambda}, \mathbf{v}-(\mathbf{V}+\xi \times \mathbf{r})\rangle_{\mathrm{P}(\mathrm{t})} \\
& \text { for all } \mathbf{v} \in \overline{\mathrm{W}}_{0}, \mathbf{V} \in \mathbf{R}^{2} \text {, and } \xi \in \mathbf{R} \text {, } \\
& \int_{\Omega} q \nabla \cdot \mathbf{u} d \mathbf{x}=0 \quad \text { for all } q \in \mathrm{L}^{2}(\Omega), \\
& \langle\boldsymbol{\mu}, \mathbf{u}-(\mathbf{U}+\omega \times \mathbf{r})\rangle_{\mathrm{P}(\mathrm{t})}=0 \quad \text { for all } \mu \in \Lambda(\mathrm{t}), \\
& \int_{\Omega}\left(\frac{\partial \mathbf{A}}{\partial \mathrm{t}}+\mathbf{u} \cdot \nabla \mathbf{A}-\mathbf{A} \cdot \nabla \mathbf{u}-\nabla \mathbf{u}^{\mathrm{T}} \cdot \mathbf{A}+\frac{1}{\lambda_{\mathrm{r}}}(\mathbf{A}-\mathbf{I})\right) \cdot \mathbf{s} \mathrm{d} \mathbf{x}=0 \quad \text { for all } \mathbf{s} \in \overline{\mathrm{W}}_{\mathrm{A} 0}, \\
& \left.\mathbf{u}\right|_{\mathrm{t}=0}=\mathbf{u}_{\mathrm{o}} \quad \text { in } \Omega, \\
& \left.\mathbf{A}\right|_{\mathrm{t}=0}=\mathbf{A}_{\mathrm{o}} \quad \text { in } \Omega,
\end{aligned}
$$


as well as the kinematic equations and the initial conditions for the particle linear and angular velocities. Here $\mathbf{F}^{\prime}$ is the additional body force applied to the particles to limit the extent of overlap (see (27) below) and $\lambda$ is the distributed Lagrange multiplier

$$
\begin{aligned}
& \overline{\mathrm{W}}_{\mathrm{u} \Gamma}=\left\{\mathbf{v} \in \mathrm{H}^{1}(\Omega)^{2} \mid \mathbf{v}=\mathbf{u}_{\Gamma}(\mathrm{t}) \quad \text { on } \quad \Gamma\right\}, \\
& \overline{\mathrm{W}}_{0}=\mathrm{H}_{0}^{1}(\Omega)^{2}, \\
& \overline{\mathrm{W}}_{\mathrm{A}}=\left\{\mathbf{A} \in \mathrm{H}^{1}(\Omega)^{3} \mid \mathbf{A}=\mathbf{A}_{\mathrm{L}}(\mathrm{t}) \text { on } \Gamma^{-}\right\}, \\
& \overline{\mathrm{W}}_{\mathrm{A} 0}=\left\{\mathbf{A} \in \mathrm{H}^{1}(\Omega)^{3} \mid \mathbf{A}=0 \quad \text { on } \quad \Gamma^{-}\right\}, \\
& \mathrm{L}_{0}^{2}(\Omega)=\left\{\mathrm{q} \in \mathrm{L}^{2}(\Omega) \mid \int_{\Omega} \mathrm{q} \mathrm{d} \mathbf{x}=0\right\},
\end{aligned}
$$

and $\Lambda(\mathrm{t})$ is $\mathrm{L}^{2}(\mathrm{P}(\mathrm{t}))^{2}$, with $\langle., .\rangle_{\mathrm{P}(\mathrm{t})}$ denoting the $\mathrm{L}^{2}$ inner product over the particle, where $\Gamma^{-}$is the upstream part of $\Gamma$. In our simulations, since the velocity and $\mu$ are in $\mathrm{L}^{2}$, we will use the following inner product

$$
\langle\boldsymbol{\mu}, \mathbf{v}\rangle_{\mathrm{P}(\mathrm{t})}=\int_{\mathrm{P}(\mathrm{t})}(\boldsymbol{\mu} \cdot \mathbf{v}) \mathrm{d} \mathbf{x} .
$$

In order to solve the above problem numerically, we will discretize the domain using a regular finite element triangulation $T_{h}$ for the velocity and configuration tensor, where $h$ is the mesh size, and a regular triangulation $\mathrm{T}_{2 \mathrm{~h}}$ for the pressure. The following finite dimensional spaces are defined for approximating $\overline{\mathrm{W}}_{\mathrm{u} \Gamma}, \overline{\mathrm{W}}_{0}, \overline{\mathrm{W}}_{\mathrm{A}}, \overline{\mathrm{W}}_{\mathrm{A} 0}, \mathrm{~L}^{2}(\Omega)$ and $\mathrm{L}_{0}^{2}(\Omega)$ :

$$
\begin{aligned}
& \mathrm{W}_{\mathrm{u} \Gamma, \mathrm{h}}=\left\{\mathbf{v}_{\mathrm{h}} \in \mathrm{C}^{0}(\bar{\Omega})^{2}\left|\mathbf{v}_{\mathrm{h}}\right|_{\mathrm{T}} \in \mathrm{P}_{1} \times \mathrm{P}_{1} \text { for all } \mathrm{T} \in \mathrm{T}_{\mathrm{h}}, \mathbf{v}_{\mathrm{h}}=\mathbf{u}_{\Gamma, \mathrm{h}} \text { on } \Gamma\right\}, \\
& \mathrm{W}_{0, \mathrm{~h}}=\left\{\mathbf{v}_{\mathrm{h}} \in \mathrm{C}^{0}(\bar{\Omega})^{2}\left|\mathbf{v}_{\mathrm{h}}\right|_{\mathrm{T}} \in \mathrm{P}_{1} \times \mathrm{P}_{1} \text { for all } \mathrm{T} \in \mathrm{T}_{\mathrm{h}}, \mathbf{v}_{\mathrm{h}}=0 \text { on } \Gamma\right\}, \\
& \mathrm{L}_{\mathrm{h}}^{2}=\left\{\mathrm{q}_{\mathrm{h}} \in \mathrm{C}^{0}(\bar{\Omega})\left|\mathrm{q}_{\mathrm{h}}\right|_{\mathrm{T}} \in \mathrm{P}_{1} \text { for all } \mathrm{T} \in \mathrm{T}_{2 \mathrm{~h}}\right\}, \\
& \mathrm{L}_{0, \mathrm{~h}}^{2}=\left\{\mathrm{q}_{\mathrm{h}} \in \mathrm{L}_{\mathrm{h}}^{2} \mid \int_{\Omega} \mathrm{q}_{\mathrm{h}} \mathrm{d} \mathbf{x}=0\right\}, \\
& \mathrm{W}_{\mathrm{Ah}}=\left\{\mathbf{s}_{\mathrm{h}} \in \mathrm{C}^{0}(\bar{\Omega})^{3}\left|\mathbf{s}_{\mathrm{h}}\right|_{\mathrm{T}} \in \mathrm{P}_{1} \times \mathrm{P}_{1} \times \mathrm{P}_{1} \text { for all } \mathrm{T} \in \mathrm{T}_{\mathrm{h}}, \mathbf{s}_{\mathrm{h}}=\mathbf{A}_{\mathrm{L}, \mathrm{h}} \text { on } \Gamma^{-}\right\}, \\
& \mathrm{W}_{\mathrm{A} 0, \mathrm{~h}}=\left\{\mathbf{s}_{\mathrm{h}} \in \mathrm{C}^{0}(\bar{\Omega})^{3}\left|\mathbf{s}_{\mathrm{h}}\right|_{\mathrm{T}} \in \mathrm{P}_{1} \times \mathrm{P}_{1} \times \mathrm{P}_{1} \text { for all } \mathrm{T} \in \mathrm{T}_{\mathrm{h}}, \mathbf{s}_{\mathrm{h}}=0 \text { on } \Gamma^{-}\right\},
\end{aligned}
$$


where $\Gamma^{-}$is the upstream part of $\Gamma$. The particle inner product terms in (14) and (16) are discretized using a triangular mesh similar to the one used in Singh et al (2000).

\subsection{Collisions and Near-Collisions}

When there is more than one particle, the right-hand side of (14) becomes a sum of particle inner product terms, one for each particle, and there is a separate equation like (16) for each particle. If two particles overlap, the problem becomes overconstrained in the overlap region. The two "copies" of (16) represent incompatible side constraints on $\mathbf{u}$. And on the right-hand side of (14), the terms corresponding to the two overlapping particles represent two supposedly independent distributed Lagrange multipliers, associated with the respective rigid-body motion constraints. But as just noted, these are constraints are incompatible.

In the finite-element discretized equations, the problem becomes overconstrained even before the particles actually overlap. Specifically, it becomes overconstrained when two particles both overlap a single velocity element. The velocity field $\mathbf{u}$ is a single linear function on this element, but the two discretized "copies" of (16) are "trying" to force it to match two different rigid motions (in the portions of the element which overlap the respective particles).

In Glowinski et al (1998) and Singh et al (2000), this situation was avoided by imposing a particle-particle repulsive force strong enough to ensure that the particle-particle gap never becomes so small that both particles intersect a single velocity element. In the modified DLM scheme described in this article we allow the particles to touch or slightly overlap, and eliminate the inconsistency in the equations by the following strategy: if each particle inner product is represented by a nonsymmetric matrix, whose rows correspond to the nodes of the particle mesh, and whose columns correspond to the nodes of the velocity mesh, then for a given particle, we "zero out" any column which corresponds to a velocity node which is closer to (the center of) another particle. In other words:

(1) If $\mathbf{v}$ is a velocity test function corresponding to a given one of the three vertices of a velocity element overlapped by two particles, we drop, from the right-hand side of (14), the particle inner product term corresponding to the particle whose center is farther from the given vertex.

(2) In the "copy" of (16) corresponding to a given particle, if $\mu$ is any particle test function for that particle's mesh and the velocity field $\mathbf{u}$ is expanded with respect to the global velocity basis functions, then we drop any nonzero terms corresponding to velocity nodes which are closer to another particle.

A little reflection will convince the reader that the resulting equations are no longer overdeterminedeven when two particles overlap — as long as the overlap is not too large. 
In our code, we impose a repulsive force when the particles overlap. In simulations, the repulsive force is assumed to be large enough so that the overlap between the particles and the walls is smaller than one hundredth of the velocity element size. The additional body force-which is repulsive in nature-is added to (8). The particle-particle repulsive force is given by

$$
\mathbf{F}_{i j}^{P}= \begin{cases}0 & \text { for } d_{i}>2 R \\ k\left(\mathbf{X}_{\mathrm{i}}-\mathbf{X}_{\mathrm{j}}\right)\left(2 R-d_{i, j}\right) & \text { for } d_{i}<2 R\end{cases}
$$

where $d_{i, j}$ is the distance between the centers of the ith and jth particles, $R$ is the particle radius which is assumed to be the same for all particles, $\mathbf{X}_{\mathrm{i}}$ and $\mathbf{X}_{\mathrm{j}}$ are the position vectors of the particle centers, and $\mathrm{k}$ is the stiffness parameter. The repulsive force between the particles and the wall is given by

$$
\mathbf{F}_{i j}^{W}= \begin{cases}0 & \text { for } d_{i}>2 R \\ k_{w}\left(\mathbf{X}_{\mathrm{i}}-\mathbf{X}_{\mathrm{j}}\right)\left(2 R-d_{i}\right) & \text { for } d_{i}<2 R\end{cases}
$$

where $d_{i}$ is the distance between the centers of the ith particle and the imaginary particle on the other side of the wall $\Gamma_{j}$, and $k_{w}$ is the stiffness parameter for particle wall collision (see Fig. 2). The above particle-particle repulsive forces and the particle-wall repulsive forces are added to (8) to obtain

$$
\mathbf{M}_{\mathrm{i}} \frac{\mathrm{d} \mathbf{U}_{\mathrm{i}}}{\mathrm{dt}}=\mathbf{M}_{\mathrm{i}} \mathbf{g}+\mathbf{F}_{\mathrm{i}}+\mathbf{F}_{\mathrm{i}}^{\prime}
$$

where

$$
\mathbf{F}_{\mathrm{i}}^{\prime}=\sum_{\substack{\mathrm{j}=1 \\ \mathrm{j} \neq \mathrm{i}}}^{\mathrm{N}} \mathbf{F}_{\mathrm{i}, \mathrm{j}}^{\mathrm{P}}+\sum_{\mathrm{j}=1}^{4} \mathbf{F}_{\mathrm{i}, \mathrm{j}}^{\mathrm{W}}
$$

is the repulsive force exerted on the ith particle by the other particles and the walls. The repulsive force acts only when the particles overlap each other.

\section{Results}

We present the results of several simulations, to demonstrate that the scheme works correctly, and that it reproduces interesting dynamical behavior. As noted earlier, due to the lubrication forces two smooth rigid particles cannot collide in the sense that the gap between them will never become zero, but it can become exceedingly small in certain situations. In numerical simulations, therefore, truncation errors may allow particles to actually touch, or even overlap.

In this paper we will assume that the acceleration due to gravity $g=981.0$ and the fluid density $\rho_{\mathrm{L}}=1.0$. We will also assume that all dimensional quantities are in the CGS units. The stiffness parameter $\mathrm{k}$ and $\mathrm{k}_{\mathrm{w}}$ in the particle-particle and particle-wall force models are equal to $10^{4}$. This value was selected to ensure that the particles do not overlap more than 0.01 times the velocity element size, 
and depends on the liquid viscosity, the density difference, the relative velocities of the particles and the acceleration due to gravity. The coordinate system used is shown in Fig. 1. The $x-$ and $y-$ components of velocity will be denoted by $u$ and $v$, respectively.

We next discuss the numerical results obtained using the above algorithm for the particleparticle interactions between sedimenting particles. The particles are suspended in Newtonian and Oldroyd-B fluids. The parameter $\mathrm{c}$ in the Oldroyd-B model will be assumed to be 7 , i.e., $\eta_{\mathrm{p}}=7 \eta_{\mathrm{s}}$.

\subsection{Newtonian Fluid}

We begin by investigating the case of two circular particles sedimenting in a channel filled with a Newtonian fluid. One of the objectives of this study is to show that the particle trajectories before collision are independent of the mesh resolution and the time step. We have used two regular triangular meshes to show that the results converge with mesh refinement. In a triangular element there are six velocity and three pressure nodes, and thus the size of the velocity elements is one half of that of the pressure elements. The particle domain is also discretized using a triangular mesh similar to the one used in Singh et al (2000). The size of velocity elements for the first mesh is 1/96, and for the second mesh is $1 / 144$. The size of particle elements for the first mesh is $1 / 70$, and for the second mesh is $1 / 125$. The number of velocity nodes and elements in the first mesh are 111,361 and 55,296, respectively. In the second mesh, there are 249,985 velocity nodes and 124,416 elements. The time step for these simulations is 0.001 or 0.0004 .

For these calculations $\eta=0.08$, and the particle diameter and density are 0.2 and 1.1 , respectively. The initial velocity distribution in the fluid, and the particle velocities are:

$$
\begin{aligned}
& \mathbf{u}_{0}=0, \\
& \mathbf{U}_{10}=\mathbf{U}_{20}=0, \\
& \omega_{10}=\omega_{10}=0 .
\end{aligned}
$$

The channel width is 2 and height is 6 . The simulations are started at $t=0$ by dropping two particles at the center of the channel at $y=5.0$ and 5.3.

It is well known that when two particles are dropped close to each other in a Newtonian fluid they interact by undergoing repeated drafting, kissing and tumbling (Fortes, Joseph, Lundgren 1987, and Joseph, Fortes, Lundgren, Singh 1987). From Fig. 3 and 4 we note that initially both particles accelerate downwards until they reach the terminal velocity at $t \approx 0.4$. From these figures we also note that the particle in the wake falls more rapidly than the particle in front, as the drag acting on it is smaller. Thus, the gap between them decreases with time and they touch or kiss each other at $\mathrm{t} \approx 0.4$. After touching each other, the particles fall together with an approximately constant velocity of 4.0 . 
The Reynolds number based on this velocity is approximately 10. The maximum overlap between the particle is less than 0.00007 , which is approximately one hundredth of the velocity element size. Also notice that when the two particles touch the y-components of their velocities become approximately equal until they tumble and subsequently separate from each other. The tumbling of particles takes place because the configuration where they are aligned parallel to the flow direction is unstable (see Fig. 5a-c).

These results are similar to those reported in Glowinski et al (1998) and Singh et al (2000) except that in both of these papers the particle surfaces were restricted to be 1.5 times the velocity element size away from each other. It is also worth noting that since the magnitude of repulsive lubrication force increases with decreasing gap between the particles, for the modified DLM method the magnitude of these force can become much larger than for the original DLM method, as the gap size is not constrained to be larger 1.5 times the velocity element size. Consequently, during the kissing phase due to the lubrication forces the relative velocity of the two particles for the modified DLM method is much smaller than for the original DLM method shown in Fig. 7 of Glowinski et al (1998). Another consequence of this that in Fig. 4 the velocity component $\mathrm{v}$ for the two particles is approximately equal for a longer time duration than in Fig. 7 of Glowinski et al (1998).

The velocity distribution around sedimenting particles is shown in Fig. 5a-c. In Fig. 5a the two particles are aligned approximately parallel to the sedimentation direction. The particles are touching each other. Fig. $5 b$ shows the velocity distribution at $t=0.625$ when the particles are beginning to tumble. The last figure in the sequence shows the velocity distribution when the particles have separated from each other. These figures show that the velocity at the nodes that are closer to the upper particle is constrained by the upper particle, and that for the nodes closer to the lower particle is constrained by the lower particle. The method therefore allows the particles to touch as well as slightly overlap each other.

In Fig. 3 and 4 we have shown the $\mathrm{x}$ and $\mathrm{y}$-components of positions and velocities as a function of time for the two meshes described above using the time steps of 0.001 and 0.0004 . From Fig. 3 we note that when the number of nodes used is approximately doubled the $\mathrm{x}$ - and $\mathrm{y}$-components of velocities and positions for both particles (marked 1 and 2 ) before collision, i.e., for $\mathrm{t}<0.4$, remain approximately the same. The results obtained for the finer mesh are denoted by $1^{\prime}$ and $2^{\prime}$. Similarly, a comparison of the curves marked ( 1 and $\left.1^{\prime}\right)$ and (2 and $\left.2^{\prime}\right)$ in Fig. 4 shows that when the time step is reduced by a factor of 2.5 the temporal evolutions of the particle velocities and trajectories do not change significantly which shows that the results for $\mathrm{t}<0.4$ are also independent of the time step. We may therefore conclude that for $\mathrm{t}<0.4$-the time duration for which the particle-particle interactions do not significantly influence the motion of the particles- the results are independent of the mesh 
resolution. The trajectories of the particles for $t>0.4$ are highly sensitive to small errors in the trajectories before the "collision," and therefore change significantly when the mesh or time step is altered. This is a form of chaos, and therefore it is impractical to refine the time step and mesh sufficiently to accurately capture the post "collision" trajectories. However, the overall qualitative behavior of the particles in the simulations is completely realistic as long as the pre-collision trajectories are accurately resolved.

\subsection{Oldroyd-B Fluid}

For all results reported in this subsection the velocity element size is $1 / 96$, and the size of the particle elements is $1 / 70$. The particle density is 1.07 and diameter is 0.2 . The fluid viscosity is 0.24 and relaxation time is 0.3 . The time step used for all results reported this subsection is 0.0001 .

When two particles sediment in a channel filled with a viscoelastic liquid the stable configuration is the one where the particles are aligned parallel to the flow direction. For these simulations the channel width is 2 and height is 6 . In Fig. 6a and b, the positions of the two particles and $\operatorname{tr} \mathbf{A}$ distributions for $\mathrm{t}=1.0$ and 3.0 are shown, where $\operatorname{tr} \mathbf{A}=\mathrm{A}_{11}+\mathrm{A}_{22}$ is the trace of the tensor $\mathbf{A}$. $\operatorname{tr} \mathbf{A}$ is a measure of the extent of polymer stretch; in the relaxed state its value is 2 and for the stretched state it is greater than 2. The particles were dropped at a distance of $0.15 \mathrm{D}$ from each other at $\mathrm{t}=0$. The Reynolds number $\mathrm{Re}=0.48$ and $\mathrm{De}=1.725$. Also note that since the velocity of the particle on the top is larger, the distance between them decreases with time. The distance decreases to approximately 0.0001D at $\mathrm{t}=1.0$. These results are similar to those reported in Fig. 9a-c of Singh et al (2000), except that the modified DLM method used here allows the particles to come closer to each other. Also notice that the distance between particles in Fig. 6 is comparable to that for experiments. From Fig. 6a-b, where we have shown the isovalues of $\operatorname{tr} \mathbf{A}$, we note that $\operatorname{tr} \mathbf{A}$ in front of the leading particle and around the particles is relatively large. This figure also shows that it takes some time for the fluid to relax back to the state of equilibrium. This gives rise to the characteristic streak lines that are indicative of the hyperbolic nature of the constitutive equation for the stress. For this case since the viscoelastic Mach number is 0.9 and the elasticity number is 3.59 , there is a strong tendency for the particles to align parallel to the falling direction. The velocity distribution around particles is shown in Fig. 6c.

When more than two particles sediment in a viscoelastic liquid the stable configuration is the one where they are aligned parallel to the sedimentation direction. To simulate this, we consider sedimentation of ten particles in a channel of width 2 and height 8 . The simulations are started at $t=0$ by placing the particles in a vertical arrangement along the channel center. The distance between the particles is $1.5 \mathrm{D}$ and the center of the first particle is at 5.0. The particles are dropped with zero linear 
and angular velocities, and the fluid is in the quiescent relaxed state. The average Deborah number is 1.73 , the average Reynolds number is 0.5 , the viscoelastic Mach number is 0.93 and the elasticity number is 3.46. The distribution of $\operatorname{tr} \mathbf{A}$ and the particle positions at $t=0.88$ is shown in Fig. 7. We note that the particles are aligned approximately parallel to the sedimentation direction and the distance between the particles of the chain is very small. Again these results are similar to those reported in Singh et al (2000), except that the particles of the chain are packed much more tightly than in Singh et al (2000). In fact, some of the particles are slightly overlapping each other. The repulsive body force is needed to ensure that the overlap is sufficiently small.

\section{Conclusions}

The modified DLM method developed in this paper allows particles to come arbitrarily close to each other, including slightly overlap each other. When conflicting rigid body motion constraints from two different particles are applicable on a velocity node, the constraint from the particle that is closer to the node is used and the other constraint is dropped. A repulsive force is applied when the particles overlap each other to limit the overlap. In our simulations, the particles are allowed to overlap as much as $1 / 100$ of the velocity element size. The modified method allows hydrodynamic forces to be fully resolved to within the tolerance of the mesh. The extra artificial force in a security zone outside the particle which are used in all other methods are thus not needed. The modified distributed Lagrange multiplier/fictitious domain method could be used to study the particle-particle interactions as well as the interactions between particles suspended in the Newtonian and Oldroyd-B fluids.

In order to validate our code we have simulated the time dependent interactions between two particles sedimenting in Newtonian and Oldroyd-B fluids. We have verified that the results are independent of the mesh resolution as well as the size of time step. Our simulations show that when particles are dropped in a channel, and the viscoelastic Mach number (M) is less than one and the elasticity number $(\mathrm{E})$ is greater than one, the particles form a chain parallel to the flow direction and the particles in the chain touch each other. The particles dropped in a Newtonian liquid undergo characteristic drafting, kissing and tumbling. The particles approximately touch each other during the kissing phase.

\section{Acknowledgements}

This work was partially supported by National Science Foundation KDI Grand Challenge grant (NSF/CTS-98-73236), the Department of Basic Energy Science at DOE and the University of Minnesota Supercomputing Institute. 


\section{References}

A. Fortes, D.D. Joseph and T. Lundgren, 1987. Nonlinear mechanics of fluidization of beds of spherical particles, J. Fluid Mech., 177, 497-483.

V. Girault, R. Glowinski and T.-W. Pan, 1999. A fictitious domain method with distributed multiplier for the Stokes problem, Applied nonlinear analysis, Kluwer Academic/Plenum Publisher, 159-174.

R. Glowinski, T.W. Pan and J. Periaux, 1997. A Lagrange multiplier/fictitious domain method for the numerical simulation of incompressible viscous flow around moving rigid bodies, C.R. Acad. Sci. Paris 324, 361-369.

R. Glowinski, T.W. Pan, T.I Hesla and D.D. Joseph, 1998. A distributed Lagrange multiplier/ fictitious domain method for particulate flows. Int. J. of Multiphase Flows, 25, 755-794.

H.H. Hu, 1996. Direct simulation of flows of solid-liquid mixtures. Int. J. Multiphase Flow, 22, $335-352$.

P.Y. Huang, J. Feng, H.H. Hu and D.D. Joseph, 1997. Direct simulation of the motion of solid particles in Couette and Poiseuille flows of viscoelastic fluids, J. Fluid Mech. 343, 73-94.

P.Y. Huang, H.H. Hu and D.D. Joseph, 1998. Direct simulation of the sedimentation of elliptic particles in Oldroyd-B fluids, J. Fluid Mech. 362, 297-325.

D.D. Joseph, A. Fortes, T. Lundgren and P. Singh, 1987. Nonlinear mechanics of fluidization of beds of spheres, cylinders, and disks in water, SIAM Advances in Multiphase Flow and Related Problems, G. Papanicolau, ed, 101-122.

L.G. Leal, 1992. Laminar Flow and Convective Transport Processes, Scaling Principles and Asymptotic Analysis, Butterworth-Heinemann, Newton, Massachusetts.

P. Singh, D.D. Joseph, T.I Hesla, R. Glowinski and T.W. Pan, 2000. Direct numerical simulation of viscoelastic particulate flows, J. of Non Newtonian Fluid Mechanics, 91, 165-188. 


\section{Figure Captions}

Figure 1. A typical rectangular domain used in our simulations; $\Gamma^{-}$is the upstream portion of $\Gamma$.

Figure 2. The imaginary particle used for computing the repulsive force acting between a particle and a wall.

Figure 3. The velocity components $\mathrm{u}$ and $\mathrm{v}$ and the positions $\mathrm{x}$ and $\mathrm{y}$ of the sedimenting circles are shown as a function of time. The size of the velocity elements is $1 / 96$, and the size of the particle elements is $1 / 70$. The time step for the curves marked 1 and 2 is 0.001 and for the curves marked 1' and 2' is 0.0004 .

Figure 4. The velocity components $\mathrm{u}$ and $\mathrm{v}$ and positions $\mathrm{x}$ and $\mathrm{y}$ of the sedimenting circles are shown as a function of time. For curves marked 1 and 2 the size of the velocity elements is 1/96 and the size of the particle elements is $1 / 70$ and for the curves marked 1' and 2' the size of the velocity elements is $1 / 144$, and the size of the particle elements is $1 / 125$. The time step is 0.0004 .

Figure 5. A magnified view of the velocity field near the particles is shown. For (a), (b) and (c), $\mathrm{t}=0.5,0.625$ and 0.878 , respectively. The size of the velocity elements is $1 / 96$, and the size of the particle elements is $1 / 70$. The time step is 0.0004 .

Figure 6 . The size of the velocity elements is $1 / 96$, and the size of the particle elements is $1 / 70$. The time step is 0.0004 . The two particles are dropped at $\mathrm{t}=0$ at $\mathrm{y}=5.0$ and $\mathrm{y}=5.3$ along the channel center. (a) Isovalues of $\operatorname{tr} \mathbf{A}$ at $t=1.0$, (b) Isovalues of $\operatorname{tr} \mathbf{A}$ at $t=3.0$, (c) The velocity field at $t=1.0$.

Figure 7. Isovalues of $\operatorname{tr} \mathbf{A}$ at $t=1.1$ for ten particles sedimenting in a channel filled with Oldroyd-B fluid is shown. The parameters are the same as in Fig. 6. 


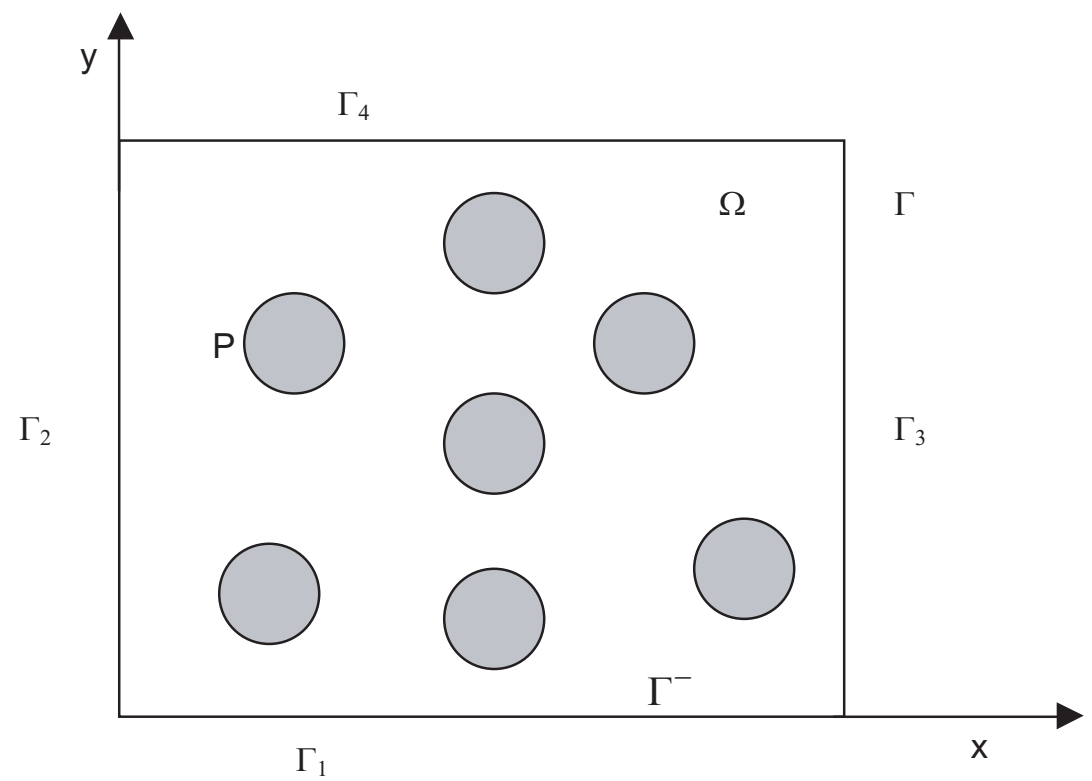




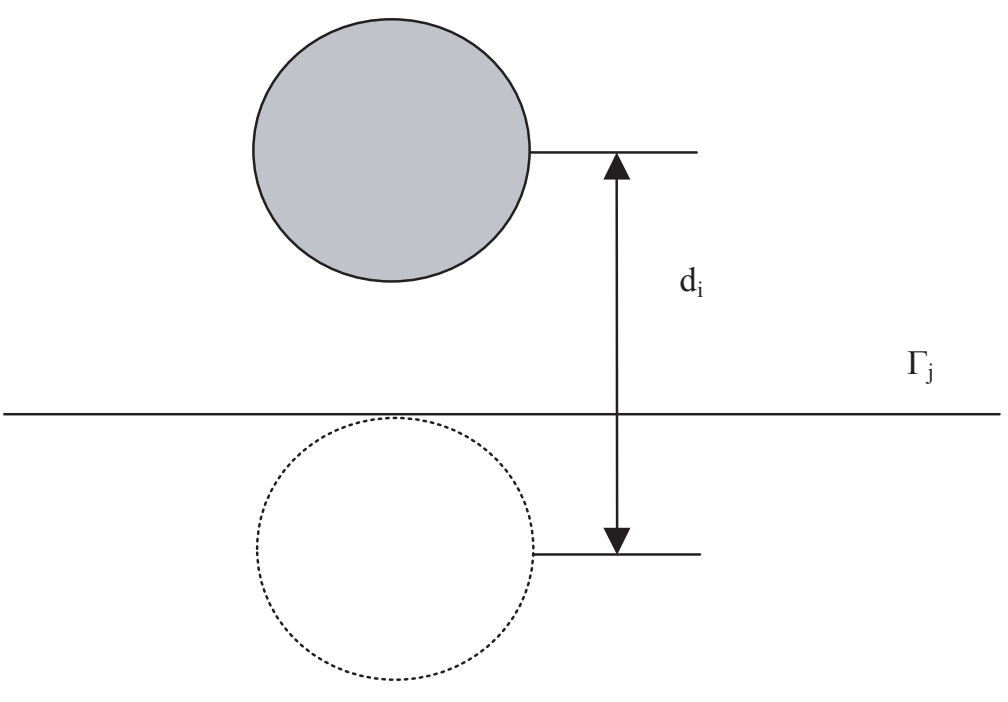




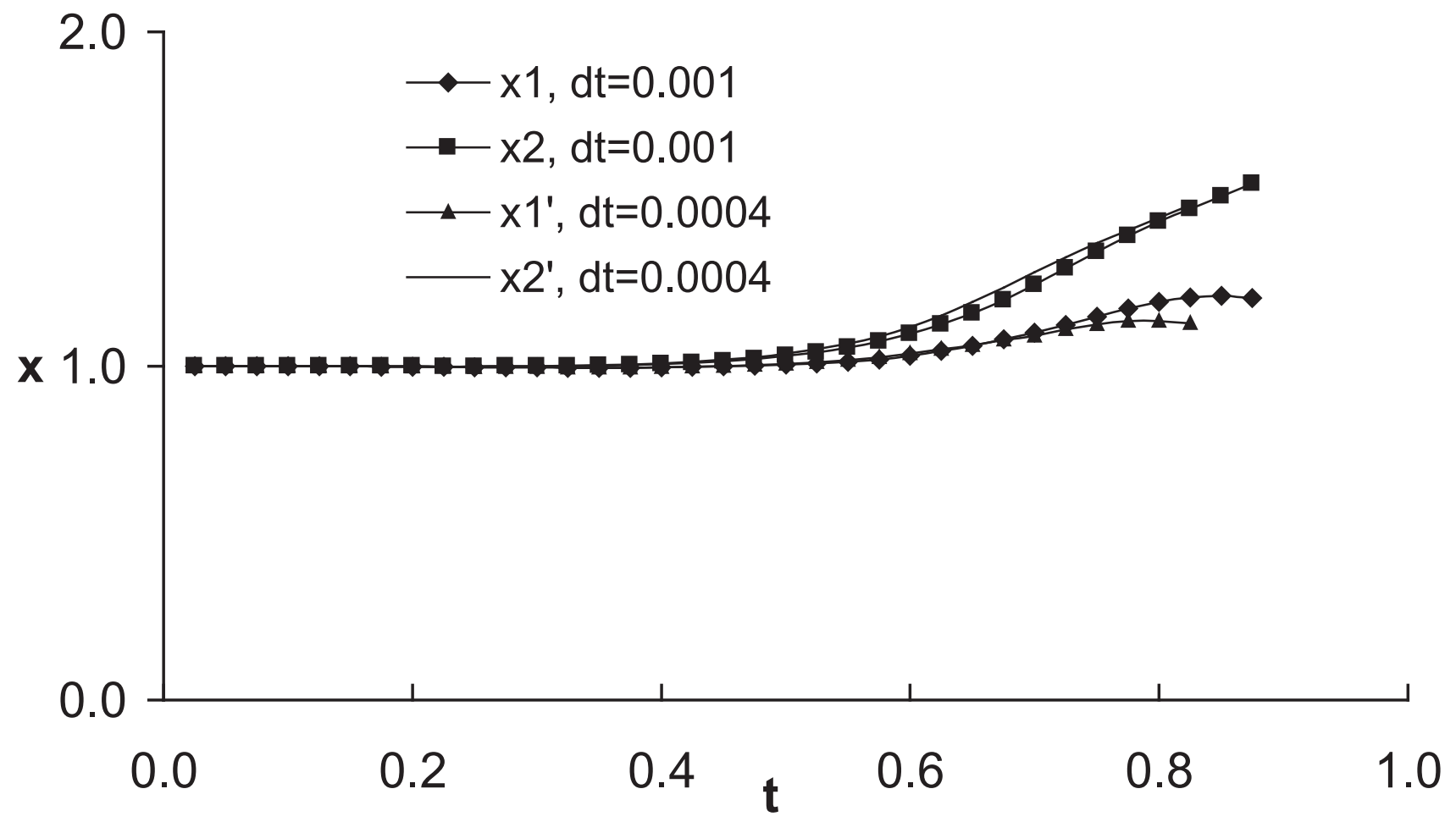




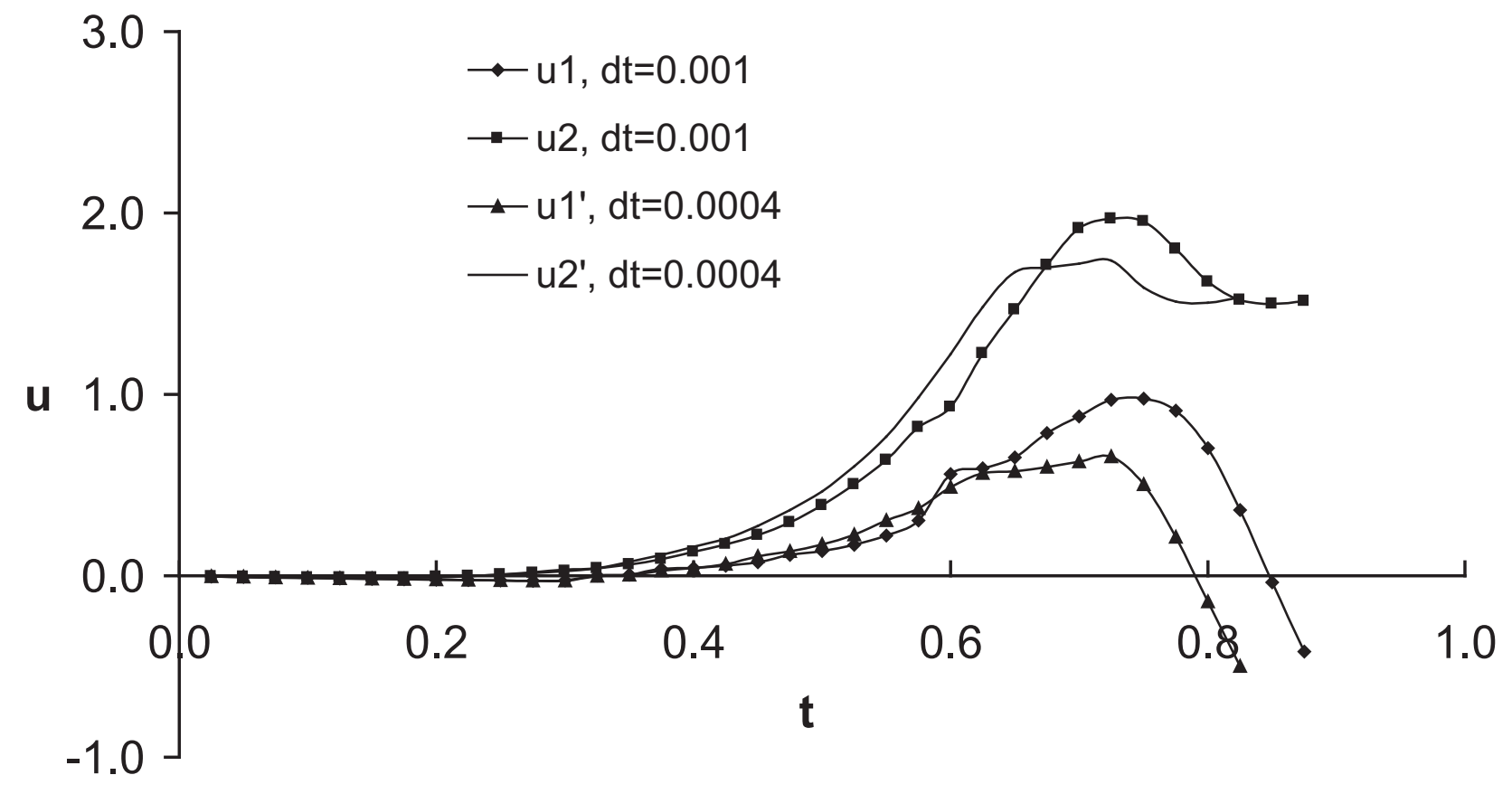




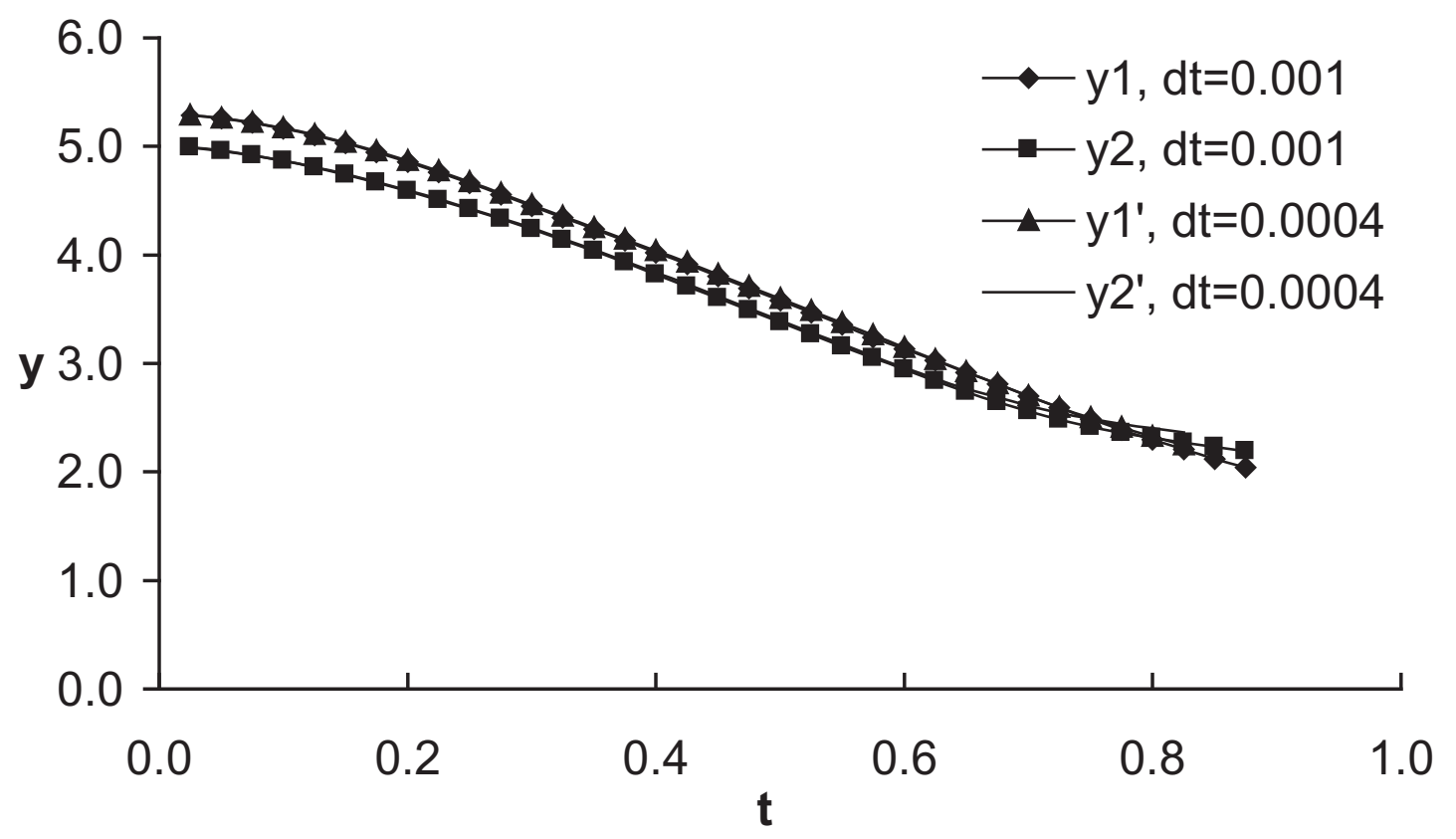




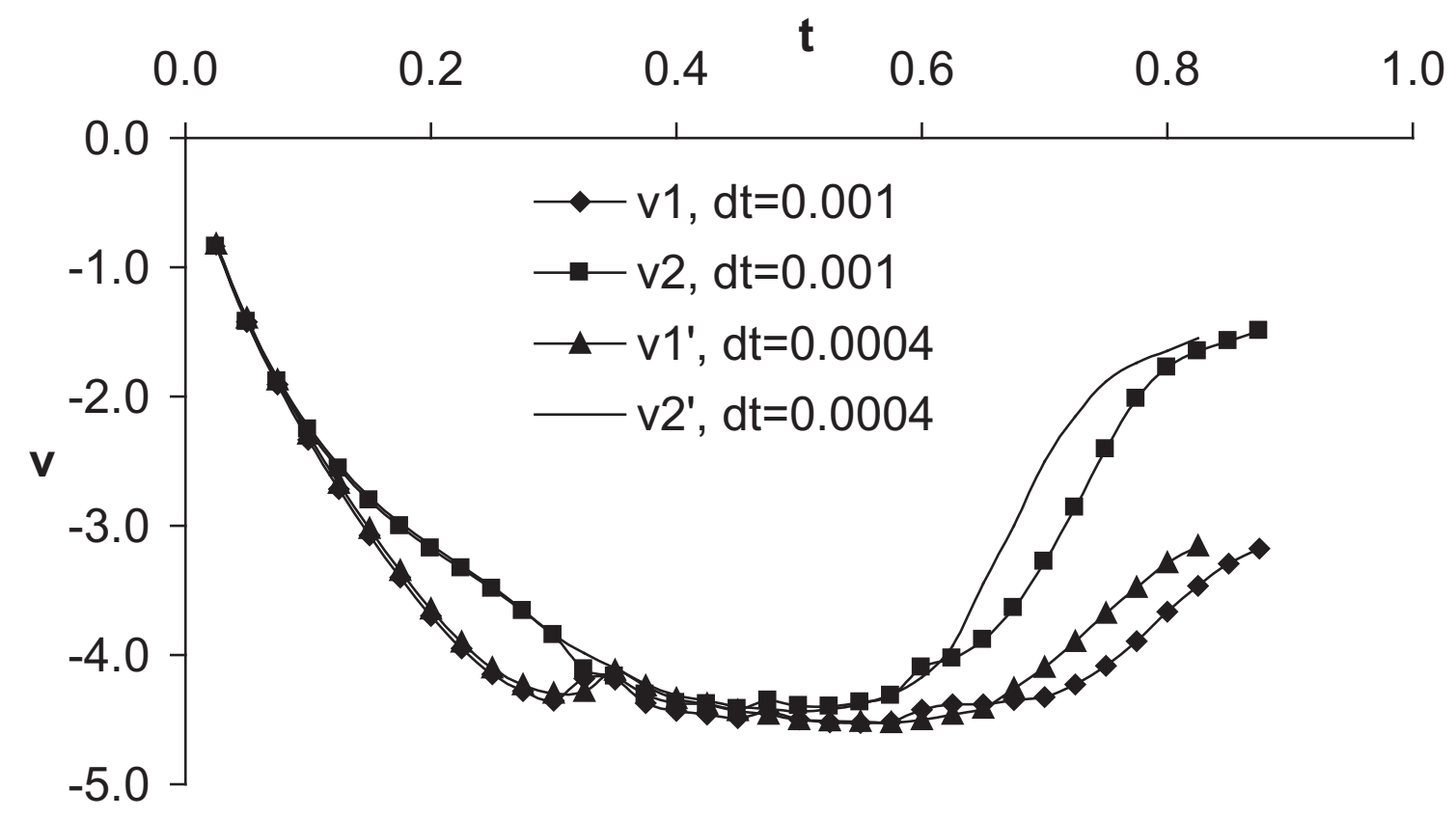




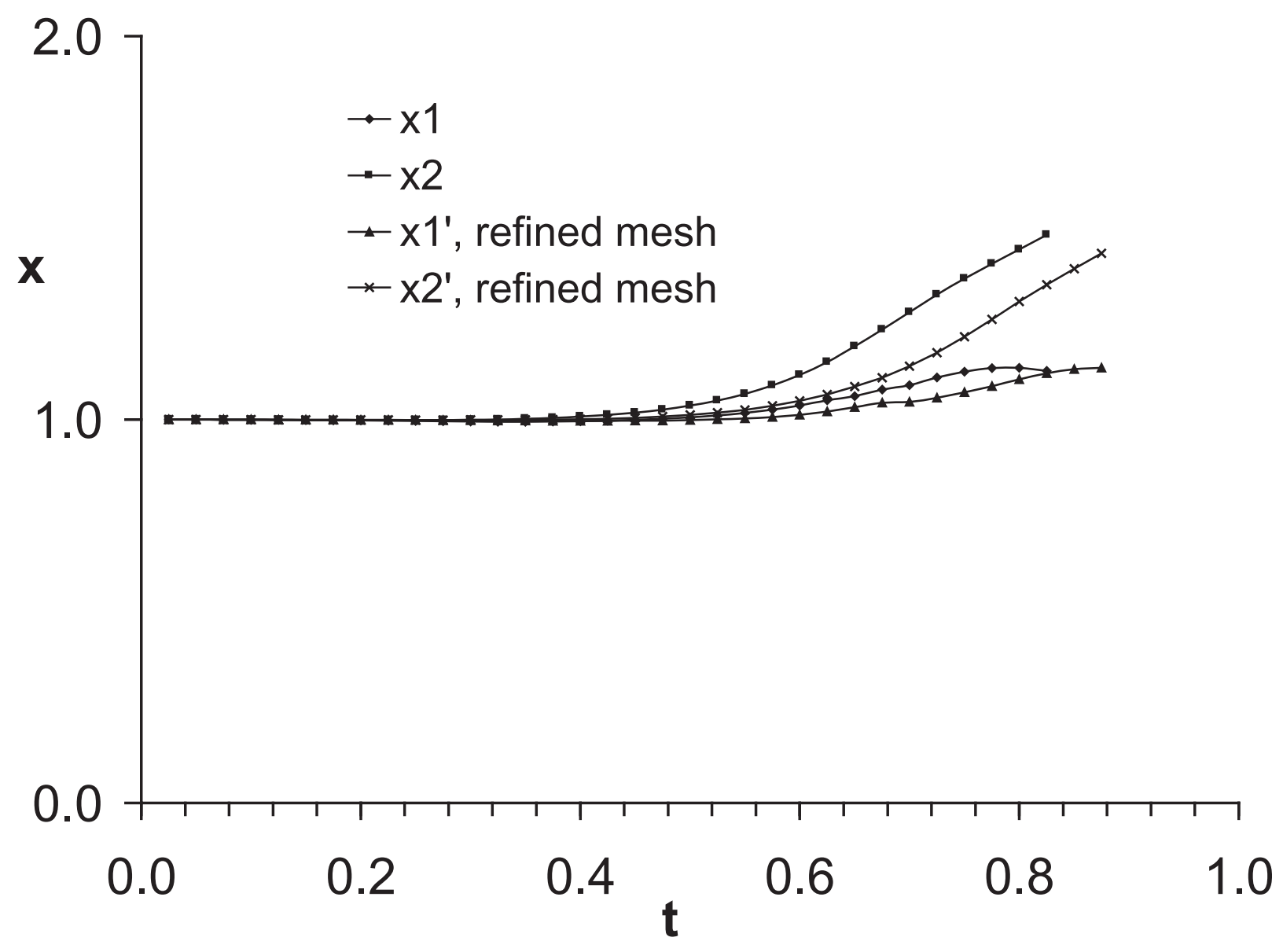




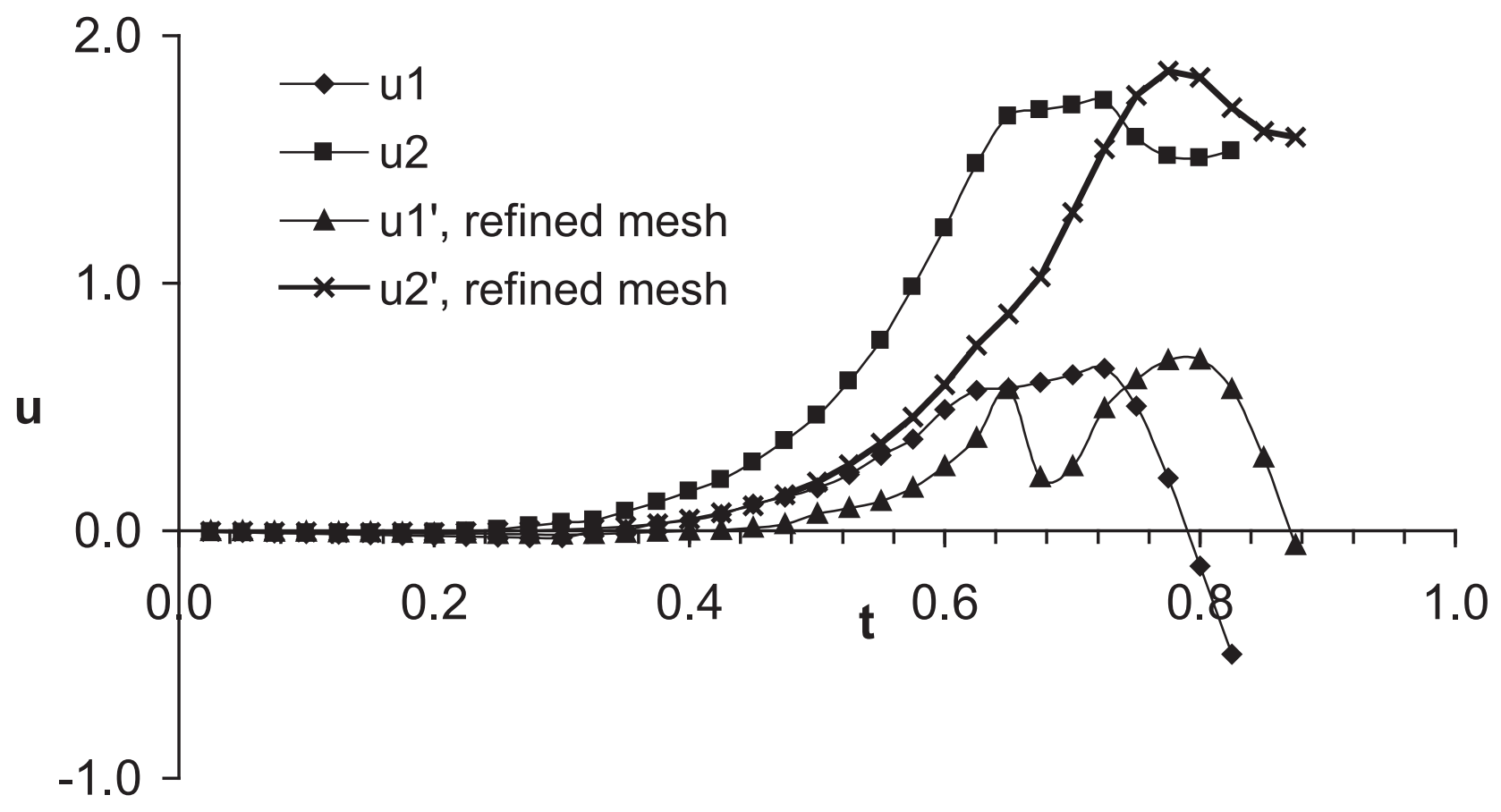




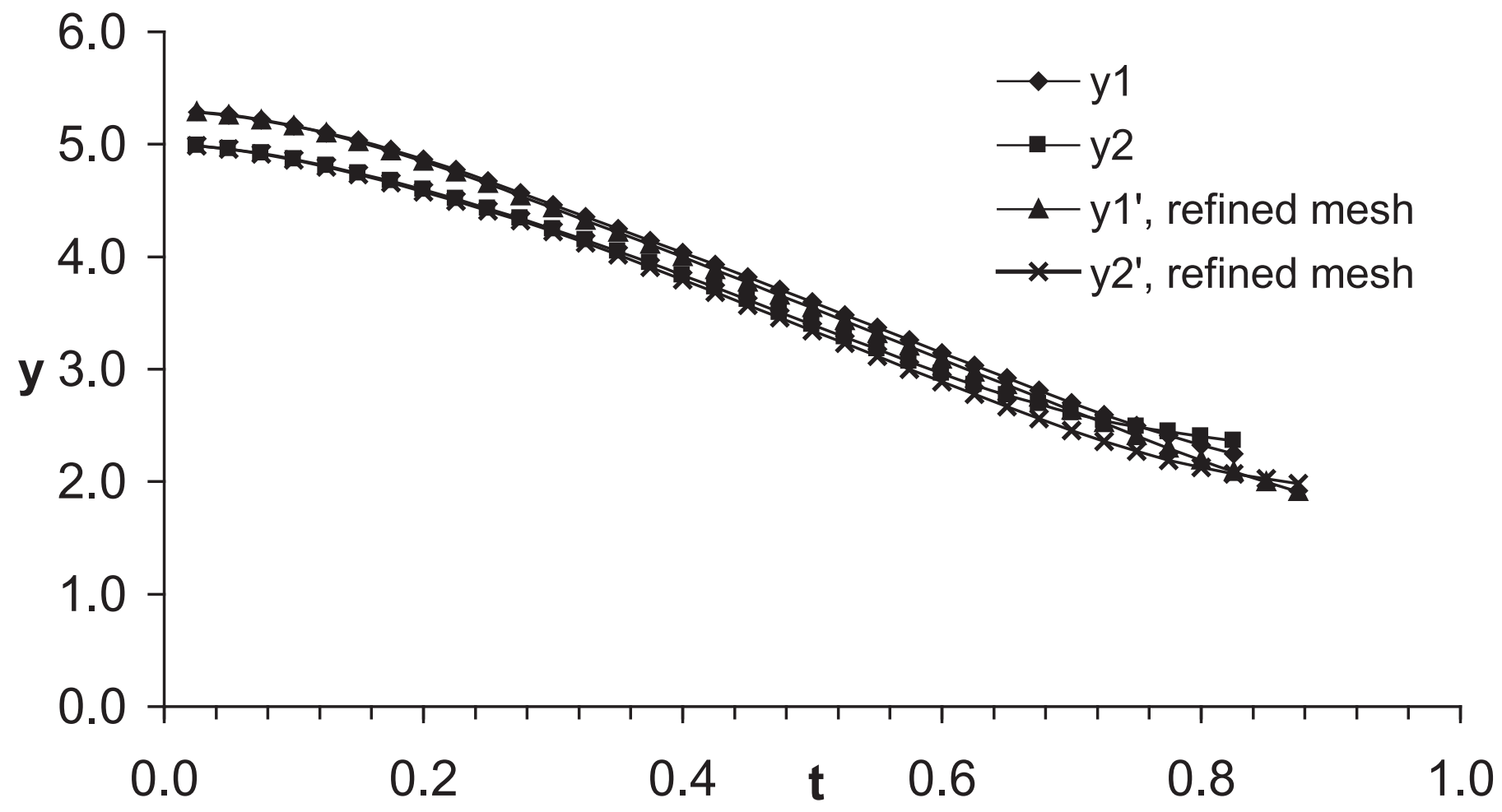




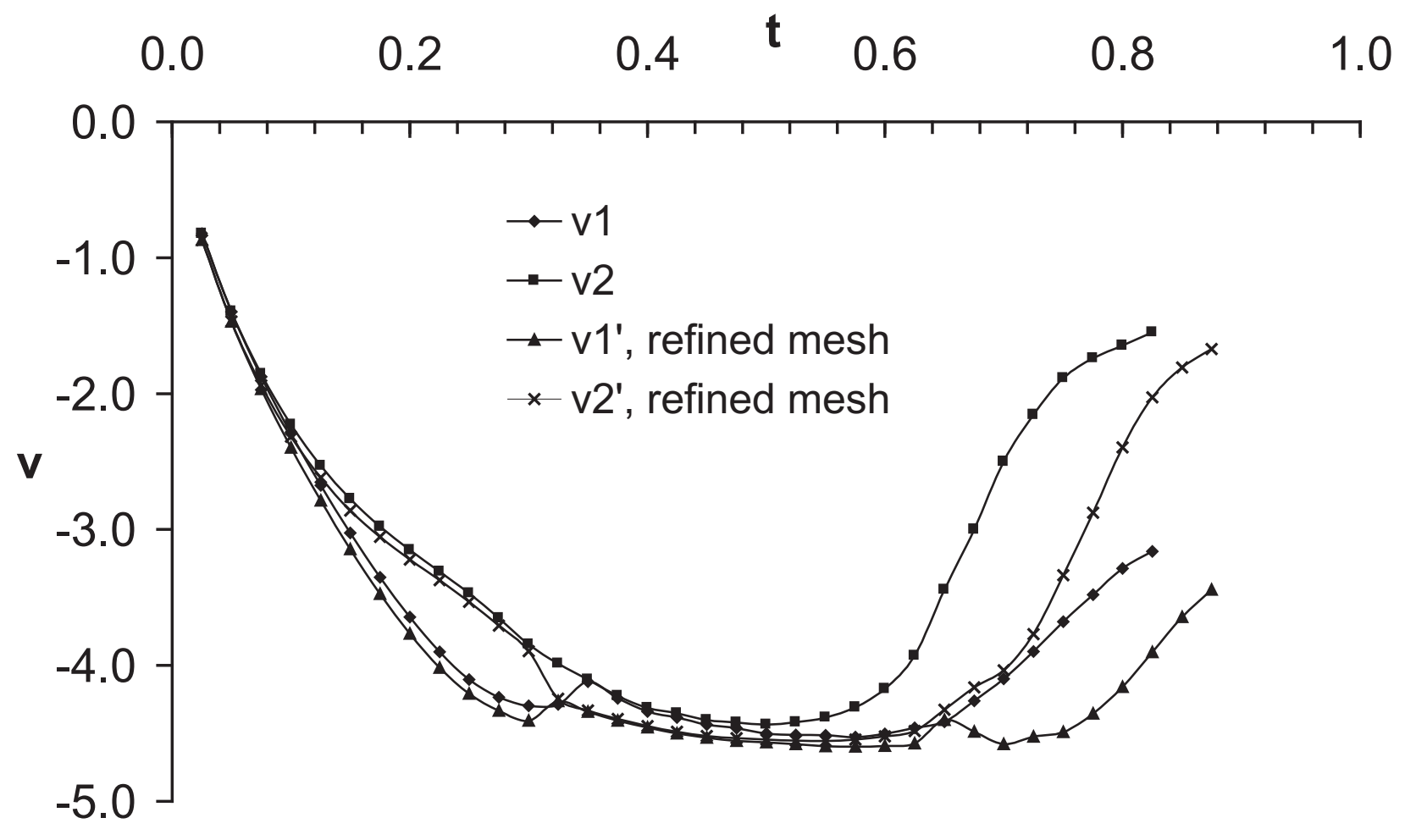




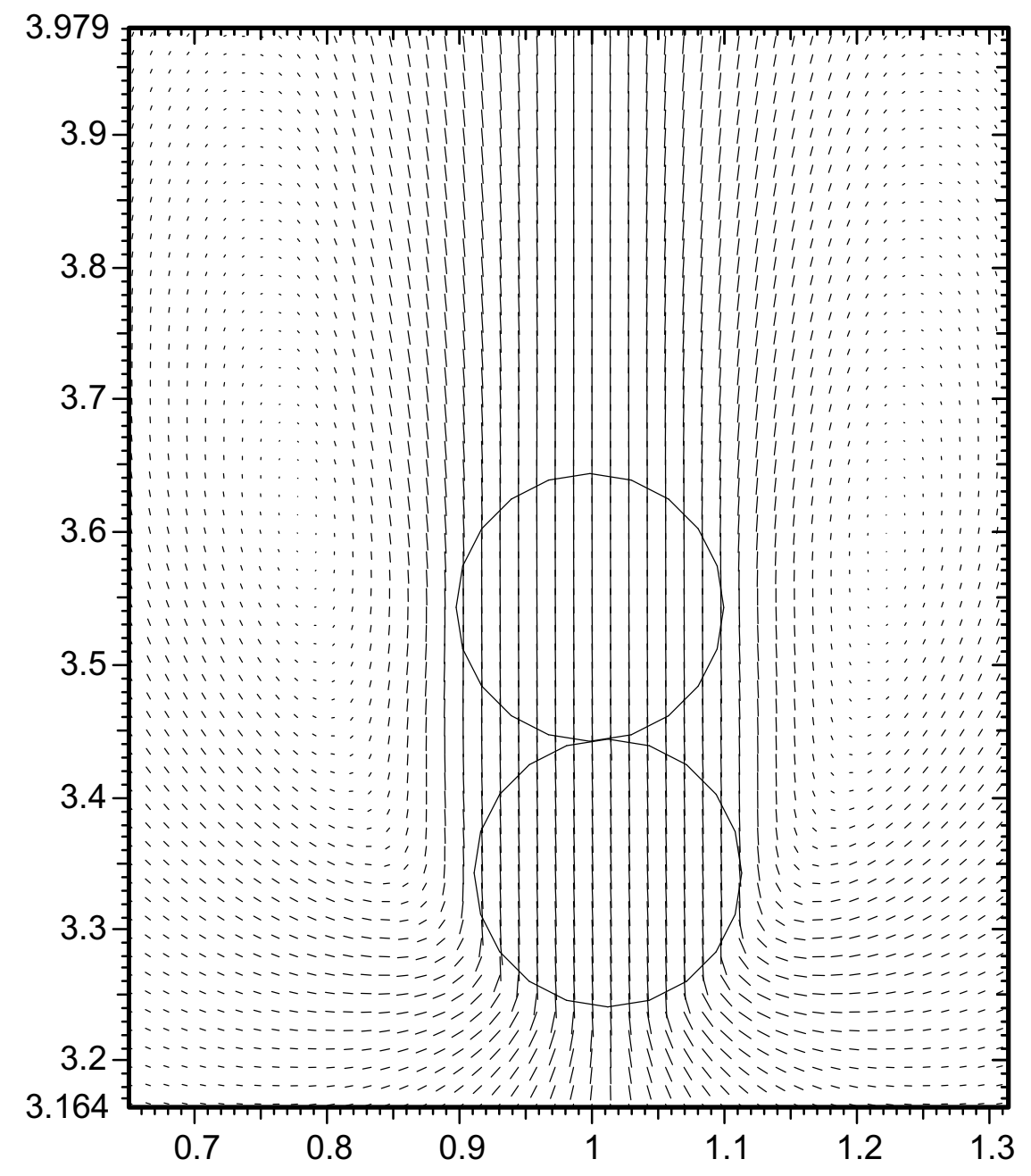




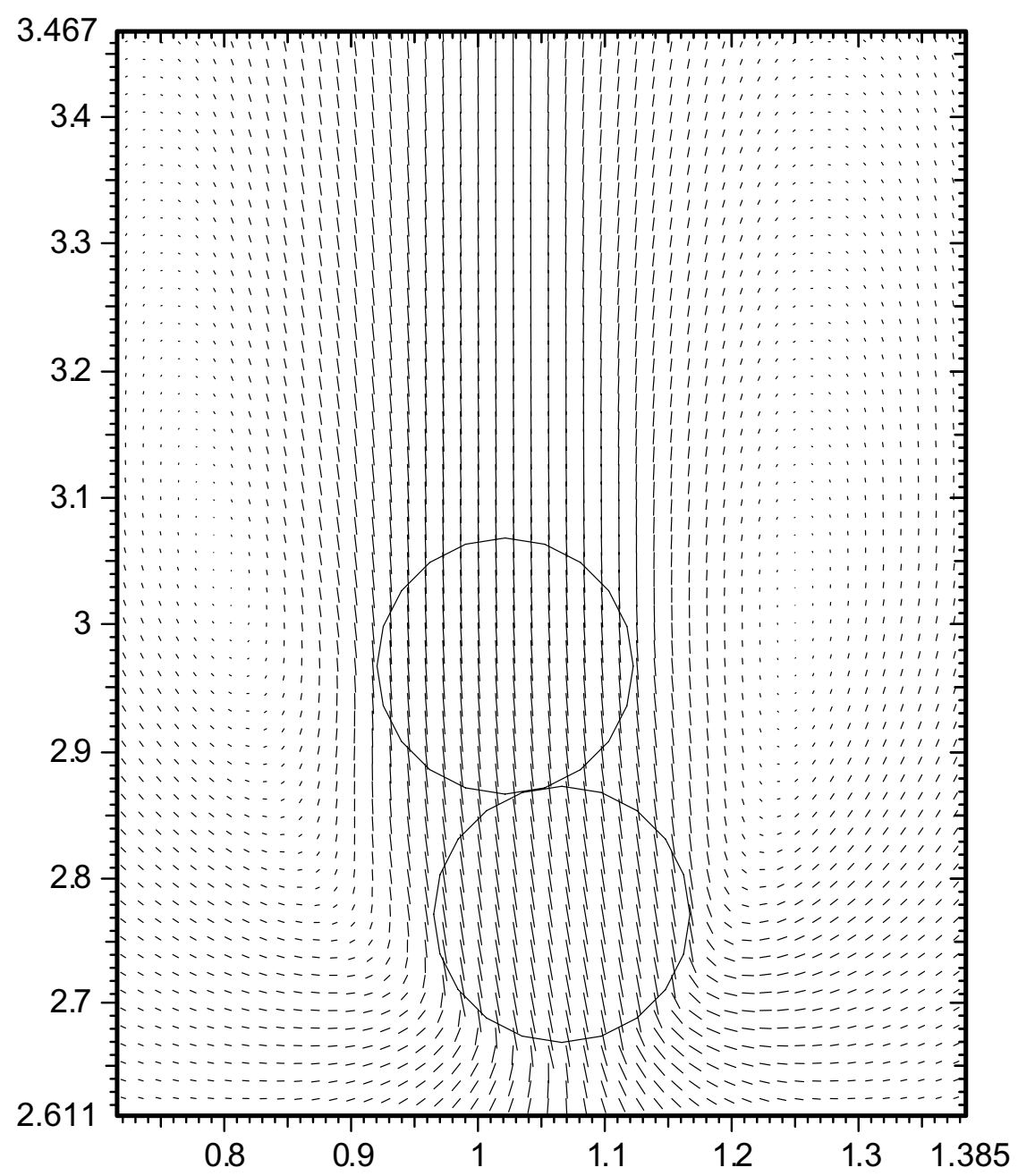




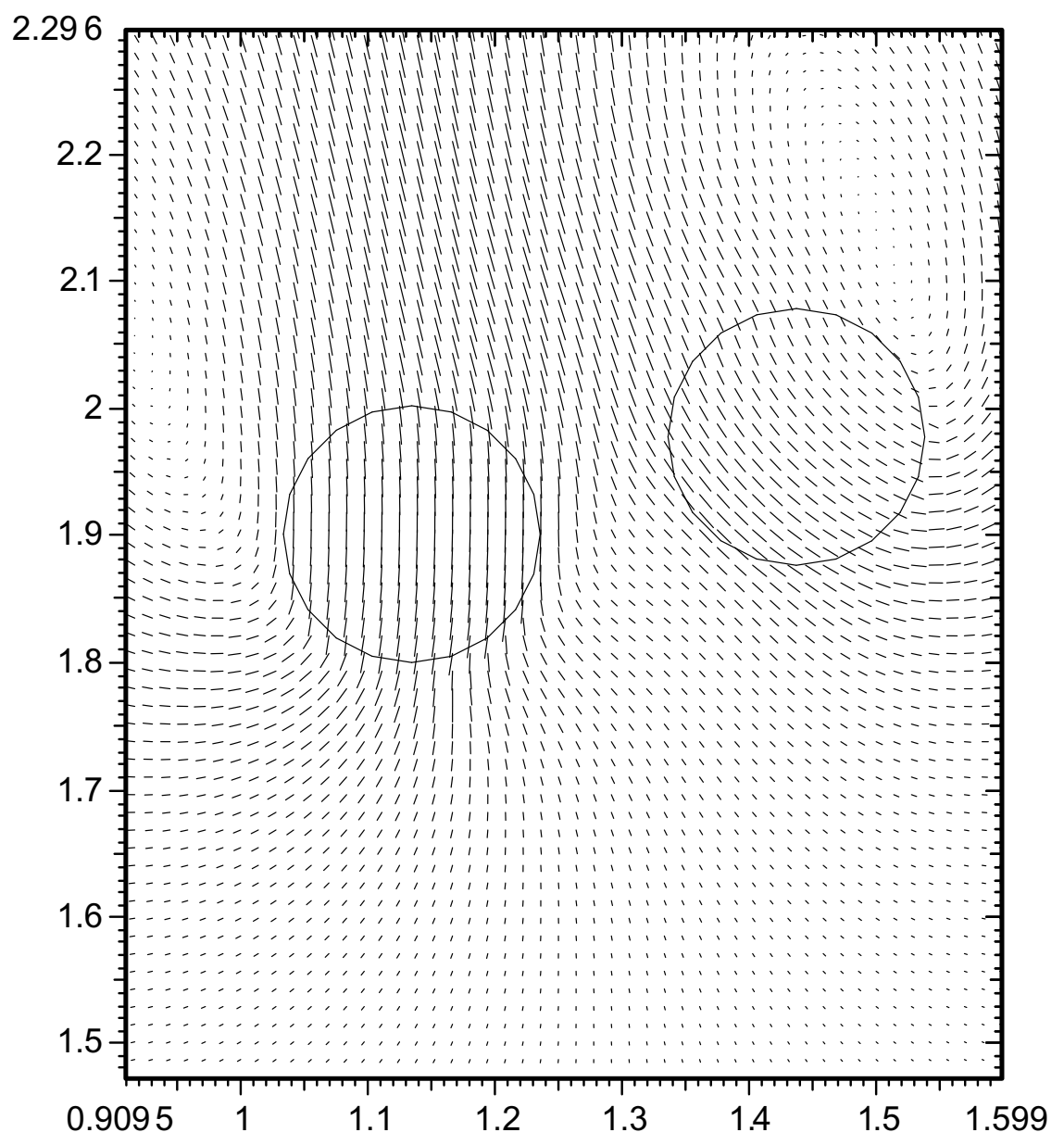




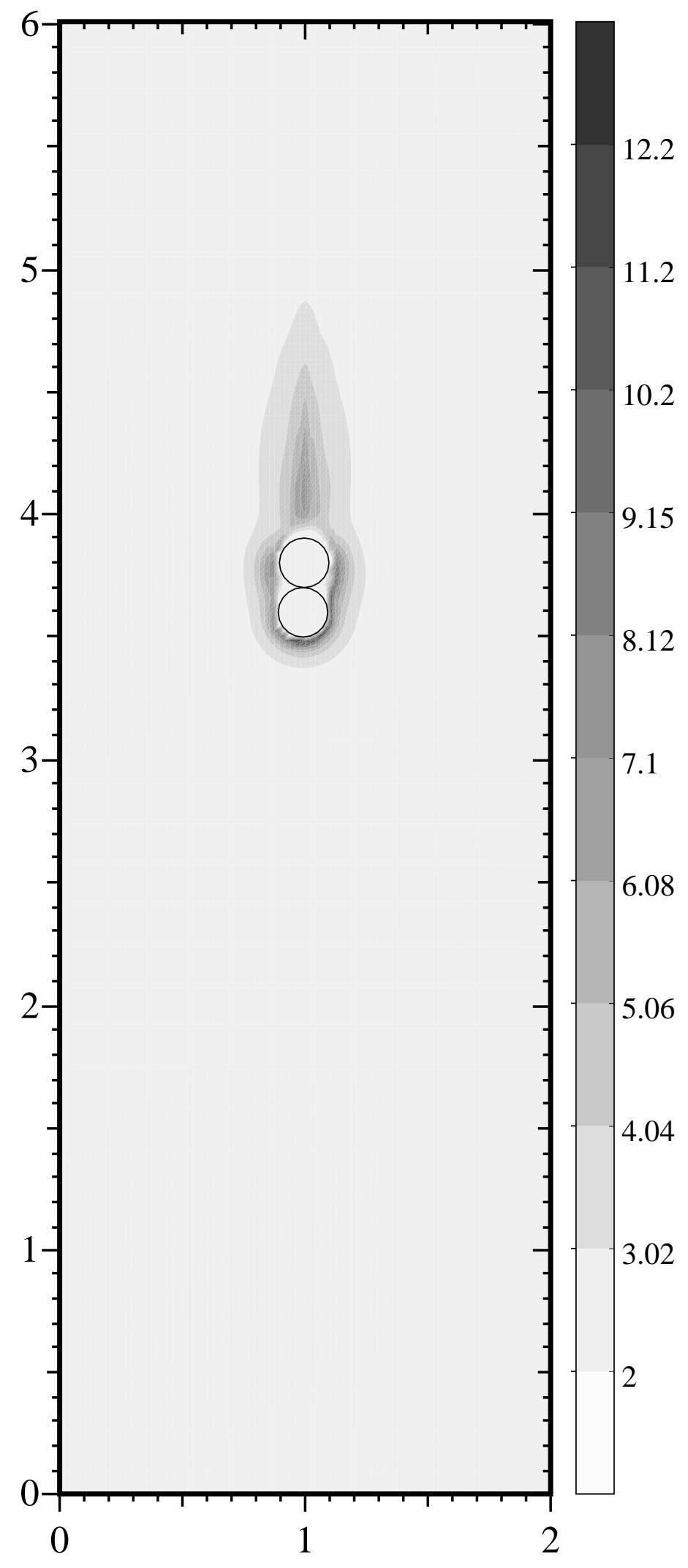




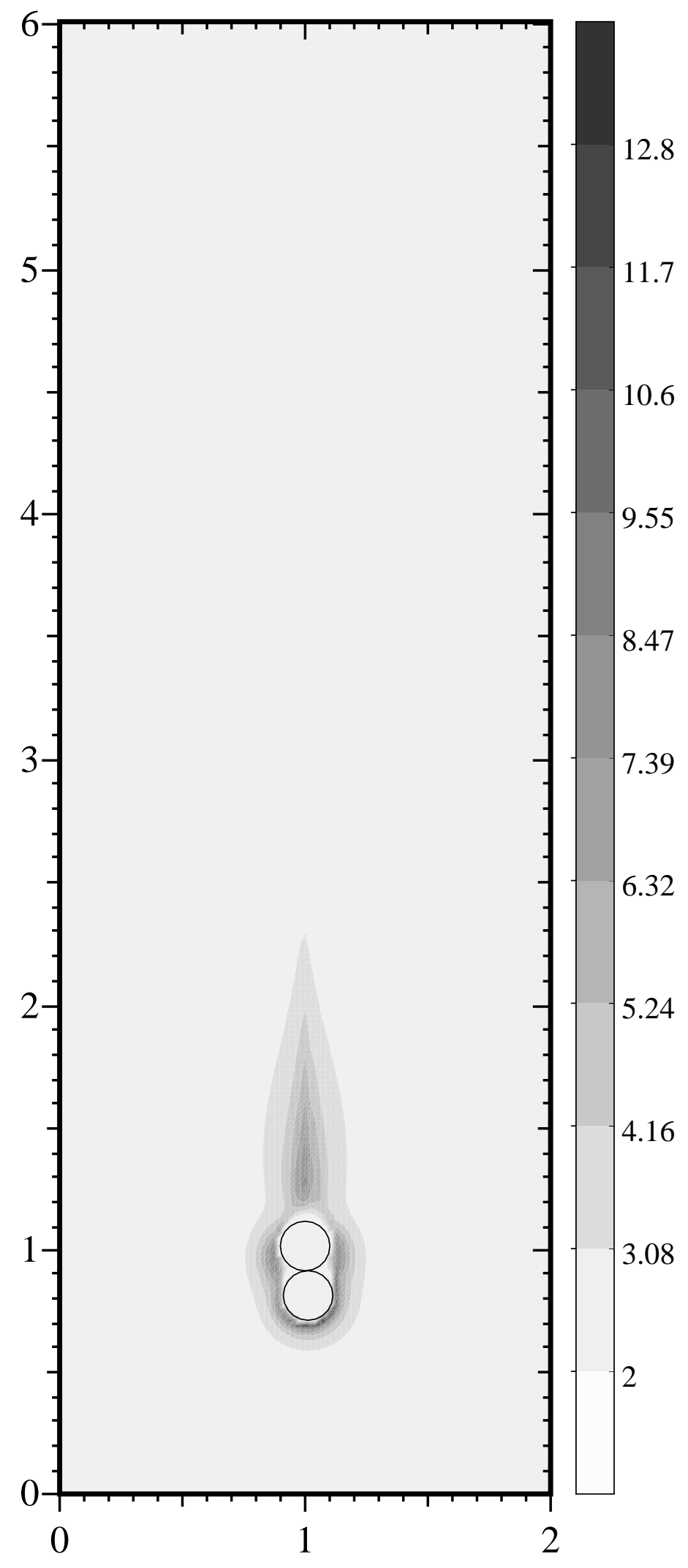




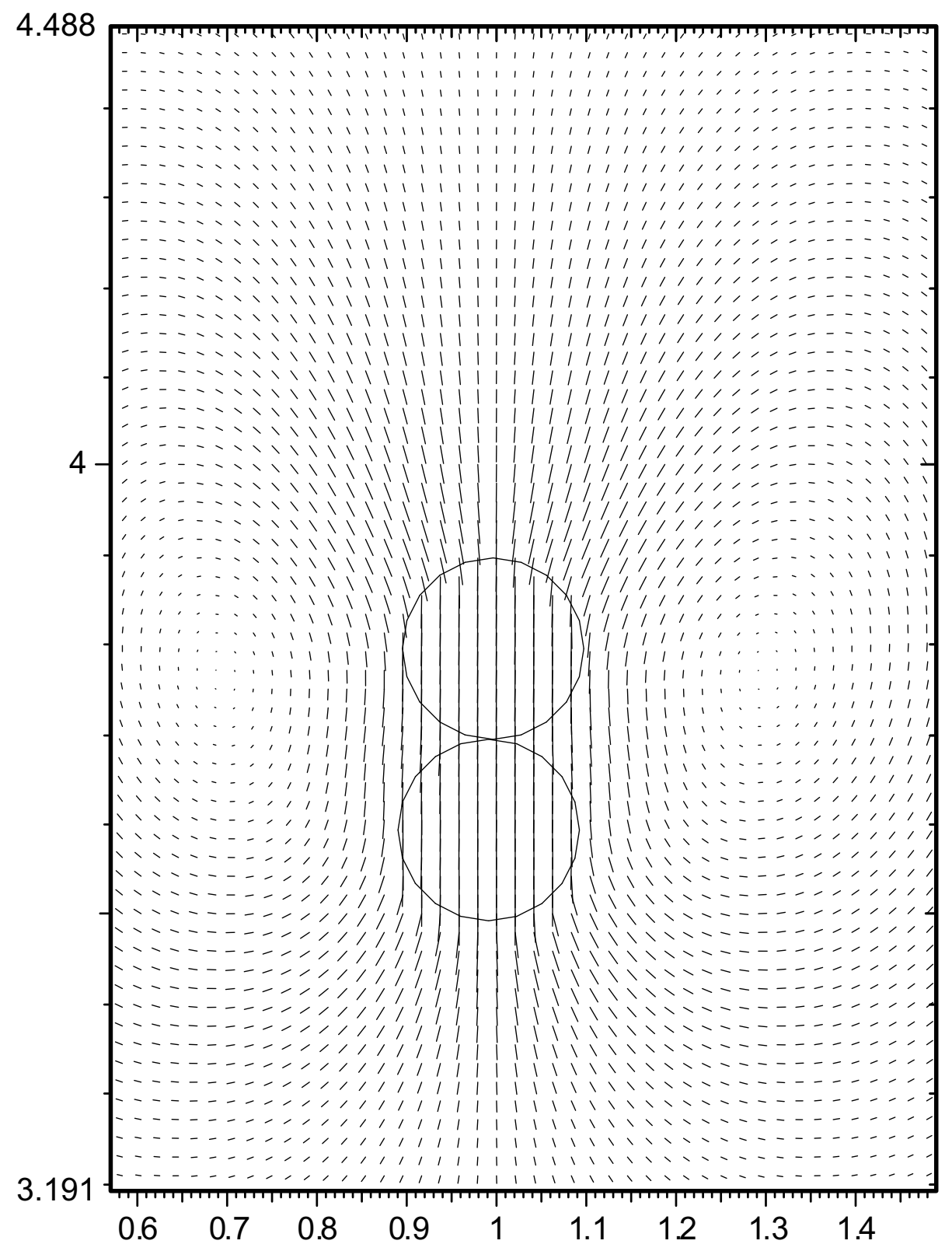




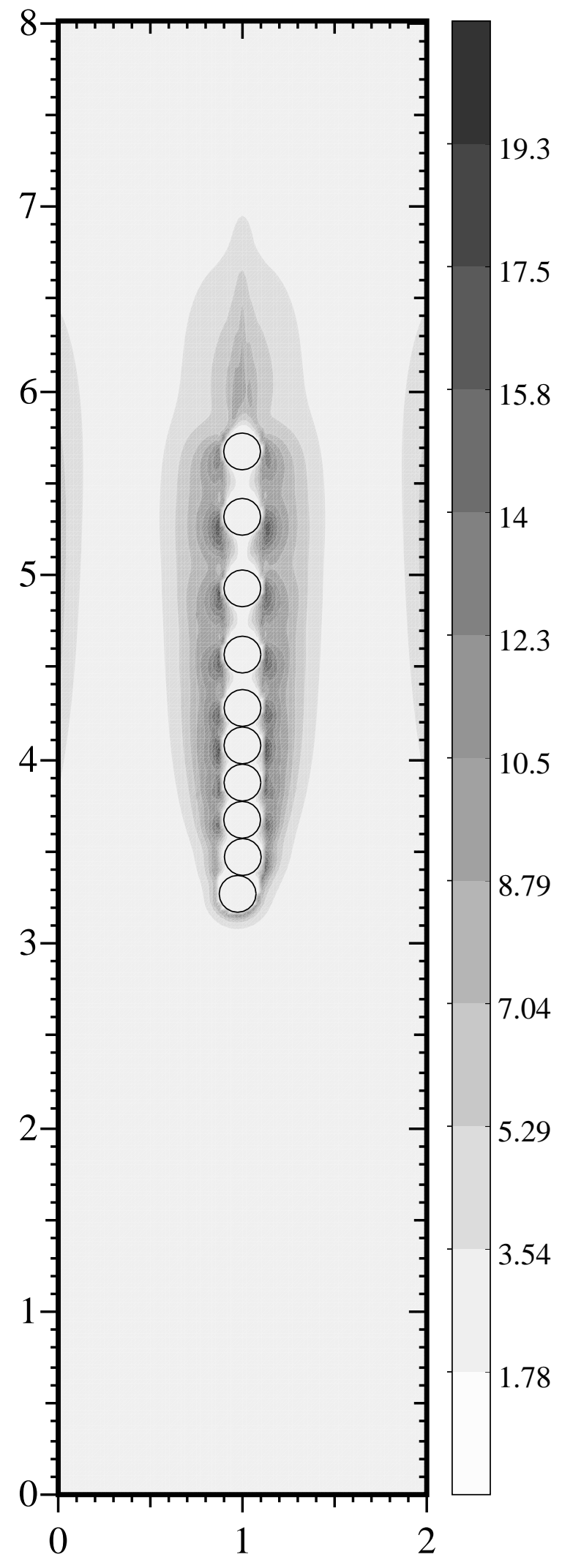

